Open J. Math. Anal., Vol. 2(2018), No. 2, pp. $78-88$

Website: https://pisrt.org/psr-press/journals/oma/

ISSN: 2616-8111 (Online) 2616-8103 (Print)

http://dx.doi.org/10.30538/psrp-oma2018.0019

\title{
OSCILLATION BEHAVIOR OF SECOND ORDER NONLINEAR DYNAMIC EQUATION WITH DAMPING ON TIME SCALES
}

\author{
FANFAN LI, ZHENLAI HAN ${ }^{1}$
}

\begin{abstract}
In this paper, we use Riccati transformation technique to establish some new oscillation criteria for the second order nonlinear dynamic equation with damping on time scales

$$
\left(r(t)\left(x^{\Delta}(t)\right)^{\alpha}\right)^{\Delta}-p(t)\left(x^{\Delta}(t)\right)^{\alpha}+q(t) f(x(t))=0 .
$$

Our results not only generalize some existing results, but also can be applied to the oscillation problems that are not covered in literature. Finally, we give some examples to illustrate our main results.
\end{abstract}

Mathematics Subject Classification: 26E70, 34C10.

Key words and phrases: Dynamic equation on time scales; Oscillation; Dynamic equation; Damped.

\section{Introduction}

The calculus theory of time scales was introduced by Hilger [1] in order to unify, extend and generalize ideas from discrete calculus, quantum calculus and continuous calculus to arbitrary time scales calculus. A time scale $\mathbb{T}$ is an arbitrary closed subset of the real numbers $\mathbb{R}$. For an introduction to time scales calculus and dynamic equations, see Bohner and Peterson books [2, 3. We are concerned with the oscillation behavior of all solutions of the second order nonlinear dynamic equation with damping on a time sceles $\mathbb{T}$ which is unbounded above

$$
\left(r(t)\left(x^{\Delta}(t)\right)^{\alpha}\right)^{\Delta}-p(t)\left(x^{\Delta}(t)\right)^{\alpha}+q(t) f(x(t))=0,
$$

Received 10-09-218. Accepted 03-11-2018.

1 Corresponding Author

(C) 2018 Fanfan Li, Zhenlai Han. This is an open access article distributed under the Creative Commons Attribution License, which permits unrestricted use, distribution, and reproduction in any medium, provided the original work is properly cited. 
where $t \in \mathbb{T}, t \geqslant t_{0}>0$. The equation will be studied under the following assumptions:

(H1) $r(t), q(t)$ are positive real-valued rd-continuous functions on $\mathbb{T}, p(t)<0$, $\frac{p(t)}{r(t)} \in \mathcal{R}^{+}$, and $\alpha$ is the quotient of two positive odd numbers;

(H2) $f: \mathbb{R} \rightarrow \mathbb{R}$ is such that $u f(u)>0$ for $u \neq 0$;

(H3) $f: \mathbb{R} \rightarrow \mathbb{R}$ is such that $f(u) \geqslant k u^{\alpha}$ for $u \neq 0$ and some $k>0$;

(H4) $\int_{t_{0}}^{\infty}\left(\frac{1}{r(t)} e_{\ominus \frac{p(s)}{r(s)}}\left(t, t_{0}\right)^{\frac{1}{\alpha}} \Delta t=\infty\right.$

We only consider these solutions of (1) which exist on some half-line $\left[t_{0}, \infty\right)_{\mathbb{T}}$ and satisfy $\sup \left\{|x(t)|: t_{1} \leqslant t<\infty\right\}>0$, for any $t_{1} \geqslant t_{0}$. If $x(t)$ satisfies (11) on $\left[t_{1}, \infty\right)_{\mathbb{T}}$ for some $t_{1} \geqslant t_{0}$, then the function $x(t)$ is called a solution of (1). A solution $x(t)$ of (1) is said to be oscillatory if it is neither eventually positive nor eventually negative, otherwise it is called nonoscillatory. The equation itself is called oscillatory if all of its solutions are oscillatory.

In the last decades, much interest has focused on obtaining sufficient conditions for the oscillation of solutions of different classes of dynamic equations on time scales, and we refer the reader to the papers [4, 5, 6, 7, 8, 9, 10, 11, 12, 13, 14. In particular, much work has been done on the following dynamic equation

$$
\begin{gathered}
\left(p(t) x^{\Delta}(t)\right)^{\Delta}+q(t) x(\sigma(t))=0, \\
\left(p(t) x^{\Delta}(t)\right)^{\Delta}+q(t)(f \circ x(\sigma(t)))=0 .
\end{gathered}
$$

Erbe et al. [15] considered the second-order nonlinear damped dynamic equation

$$
\left(r(t)\left(x^{\Delta}(t)\right)^{\gamma}\right)^{\Delta}+p(t)\left(x^{\Delta \sigma}(t)\right)^{\gamma}+q(t) f(x(\tau(t)))=0,
$$

and obtained some oscillation criteria.

Saker et al. [16] studied the oscillation criteria for difference equations with damping terms

$$
\Delta\left(a_{n}\left(\Delta x_{n}\right)^{\gamma}\right)+p_{n}\left(\Delta x_{n}\right)^{\gamma}+q_{n} f\left(x_{n+1}\right)=0,
$$

and obtained some oscillation criteria.

Deng et al. 17. researched oscillation criteria for second order nonlinear delay dynamic equations

$$
\left(r(t)\left|x^{\Delta}(t)\right|^{\gamma-1} x^{\Delta}(t)\right)^{\Delta}+p(t) f(x(\tau(t)))=0,
$$

Agwo et al. 18 considered the oscillation criteria of second order half linear delay dynamic equation

$$
\left(r(t) g\left(x^{\Delta}(t)\right)\right)^{\Delta}+p(t) f(x(\tau(t)))=0,
$$

and obtained some oscillation criteria.

Note that in the special case when $\mathbb{T}=\mathbb{R}$, (1) becomes the second-order nonlinear damped differential equation

$$
\left(r(t)\left(x^{\prime}(t)\right)^{\alpha}\right)^{\prime}+p(t)\left(x^{\prime}(t)\right)^{\alpha}+q(t) f(x(t))=0, \quad t \in \mathbb{R},
$$


and when $\mathbb{T}=\mathbb{Z}$, (1) becomes the second-order nonlinear damped difference equation

$$
\Delta\left(r(t)(\Delta x(t))^{\alpha}\right)+p(t)(\Delta x(t))^{\alpha}+q(t) f(x(t))=0, \quad t \in \mathbb{Z},
$$

where $\Delta x(t)=x(t+1)-x(t)$.

In this paper, we replace $e_{p}(t, s)$ with $e_{\ominus p}(t, s)$ and this is difference between our paper and other articles. Our result extend and improve some well-known oscillation results. The paper is organized as follows. In Section 2, we present some basic definitions and useful results from the theory of calculus on time scales on which we rely in the later section. In section 3, we intend to use the Riccati transformation technique, integral averaging technique, and inequalities to obtain some sufficient conditions for oscillation for oscillation of every solution of (1). In section 4, we give a example to illustrate our results. The last section is devoted to remarks and comments concerning our results. We also formulate possible new research directions.

\section{Preliminaries}

Lemma 2.1. 2] We say that a function $p: \mathbb{T} \rightarrow \mathbb{R}$ is regressive provided

$$
1+\mu(t) p(t) \neq 0
$$

for all $t \in \mathbb{T}^{\kappa}$ holds.

We define the set $\mathcal{R}^{+}$of all positively regressive elements of $\mathcal{R}$ by

$$
\mathcal{R}^{+}=\{p \in \mathcal{R}: 1+\mu(t) p(t)>0 \text { for all } t \in \mathbb{T}\} .
$$

$\mathcal{R}^{+}$is a subgroup of $\mathcal{R}$.

Lemma 2.2. 2] Show that if $p \in \mathcal{R}$, then the function $\ominus p$ defined by

$$
(\ominus p)(t):=-\frac{p(t)}{1+\mu(t) p(t)}
$$

for all $t \in \mathbb{T}^{\kappa}$ are also elements of $\mathcal{R}$. Since $p \in \mathcal{R}^{+}$, we have $\ominus p \in \mathcal{R}^{+}$by Lemma 2.1.

If $p \in \mathcal{R}^{+}$, then $e_{p}\left(t, t_{0}\right)>0$ for all $t \in \mathbb{T}$.

Lemma 2.3. 2] If $p \in \mathcal{R}$, then we have define the exponential function by

$$
e_{p}(t, s)=\exp \left(\int_{s}^{t} \xi_{\mu(\tau)}(p(\tau)) \Delta \tau\right) \quad \text { for } s, t \in \mathbb{T},
$$

where $\xi_{h}(z)=\frac{1}{h} \log (1+z h), h>0$.

Lemma 2.4. 2] If $p, q \in \mathcal{R}$, then

(1) $e_{p}(t, t) \equiv 1$

(2) $e_{p}(\sigma(t), s)=(1+\mu(t) p(t)) e_{p}(t, s)$;

(3) $\frac{1}{e_{p}(t, s)}=e_{\ominus p}(t, s) \cdot x$ 
Lemma 2.5. 2] Let $y \in C_{r d}$ and $p \in \mathcal{R}^{+}$, we have

$$
\begin{aligned}
\left(y e_{\ominus p}\left(\cdot, t_{0}\right)\right)^{\Delta}(t) & =y^{\Delta}(t) e_{\ominus p}(\sigma(t), t)+y(t)(\ominus p)(t) e_{\ominus p}\left(t, t_{0}\right) \\
& =y^{\Delta}(t) e_{\ominus p}(\sigma(t), t)+y(t) \frac{(\ominus p)(t)}{1+\mu(t)(\ominus p)(t)} e_{\ominus p}(\sigma(t), t) \\
& =\left(y^{\Delta}(t)-(\ominus(\ominus p))(t) y(t)\right) e_{\ominus p}(\sigma(t), t) \\
& =\left(y^{\Delta}(t)-p(t) y(t)\right) e_{\ominus p}(\sigma(t), t) .
\end{aligned}
$$

Lemma 2.6. 3] Assume that $\alpha>0$ is the ratio of positive odd integers and $x^{\alpha}(t) \in C_{r d}^{1}(I, \mathbb{R})$. Then

$$
\left(x^{\alpha}(t)\right)^{\Delta} \geqslant \begin{cases}\alpha(x(\sigma(t)))^{\alpha-1} y^{\Delta}(t), & 0<\alpha \leqslant 1, \\ \alpha(x(t))^{\alpha-1} x^{\Delta}(t), & \alpha \geqslant 1 .\end{cases}
$$

\section{Main Results}

Now, we are in a position to state and prove some new results which guarantee that every solution of (1) oscillates.

Theorem 3.1. Assume that (H1)-(H4) hold. Furthermore, assume that there exists a positive real $r d$-continuous differentiable function $v(t)$ such that

$$
\limsup _{t \rightarrow \infty} \int_{t_{0}}^{t}\left(k v(s) q(s)-\frac{\psi^{\alpha+1}(s) r(s)}{(\alpha+1)^{\alpha+1} v^{\alpha}(s)}\right) \Delta s=\infty
$$

where

$$
\psi(t)=\frac{r(t) v^{\Delta}(t)+v(t) p(t)}{r(t)}
$$

Then every solution of (1) is oscillatory.

Proof. Suppose to the contrary that $x(t)$ is a nonoscillatory solution of (1). With loss of generality, we may assume that $x(t)>0$ for $t \geqslant t_{1}>t_{0}$. We shall consider only this cases, since in view of (H2), the proof of the case when $x(t)$ is eventually negative is similar. Now, we claim that $x^{\Delta}(t)$ has a fixed sign on the interval $\left[t_{2}, \infty\right)$ for some $t_{2} \geqslant t_{1}$. From (1), since $q(t)>0$ and $f(x(t))>0$, we have

$$
\left(r(t)\left(x^{\Delta}(t)\right)^{\alpha}\right)^{\Delta}-p(t)\left(x^{\Delta}(t)\right)^{\alpha}=-q(t) f(x(t))<0,
$$

i.e.,

$$
\left(r(t)\left(x^{\Delta}(t)\right)^{\alpha}\right)^{\Delta}-p(t)\left(x^{\Delta}(t)\right)^{\alpha}<0 .
$$

By setting $y(t)=r(t)\left(x^{\Delta}(t)\right)^{\alpha}$, we immediately see that $y^{\Delta}(t)-\frac{p(t)}{r(t)} y(t)<0$, by Lemma 2.2 and Lemma 2.3, we have $\left(y(t) e_{\ominus \frac{p}{r}}\right)^{\Delta}<0$. Then $y(t) e_{\ominus \frac{p}{r}}$ is decreasing and thus $y(t)$ is eventually of one sign. Then $x^{\Delta}(t)$ has a fixed sign for all sufficiently large $t$ and we have one of the following:

$$
\left\{\begin{array}{l}
\text { Case (1). } x^{\Delta}(t) \text { is eventually positive. } \\
\text { Case }(2) \cdot x^{\Delta}(t) \text { is eventually negative. }
\end{array}\right.
$$


First, we consider Case (1). $x^{\Delta}(t)>0$ on $\left[t_{2}, \infty\right)$ for some $t_{2} \geqslant t_{1}$. Then in view (11) and (H1) we have

$$
x(t)>0, x^{\Delta}(t)>0,\left(r(t)\left(x^{\Delta}(t)\right)^{\alpha}\right)^{\Delta}<0, t \geqslant t_{2} .
$$

We see that for $t \geqslant t_{3}=\sigma\left(t_{2}\right)$

$$
r(t)\left(x^{\Delta}(t)\right)^{\alpha}>r(\sigma(t))\left(x^{\Delta}(\sigma(t))\right)^{\alpha}, x^{\alpha}(\sigma(t))>x^{\alpha}(t) .
$$

Define the function $w(t)$ by the Riccati substitution

$$
w(t):=v(t) r(t)\left(\frac{x^{\Delta}(t)}{x(t)}\right)^{\alpha}, t \geqslant t_{2} .
$$

In view of (1), we have

$$
\begin{aligned}
w^{\Delta}(t)= & r(\sigma(t))\left(x^{\Delta}(\sigma(t))\right)^{\alpha}\left(\frac{v(t)}{x^{\alpha}(t)}\right)^{\Delta}+\frac{v(t)\left(r(t)\left(x^{\Delta}(t)\right)^{\alpha}\right)^{\Delta}}{x^{\alpha}(t)} \\
= & r(\sigma(t))\left(x^{\Delta}(\sigma(t))\right)^{\alpha} \frac{v^{\Delta}(t) x^{\alpha}(t)-v(t)\left(x^{\alpha}(t)\right)^{\Delta}}{x^{\alpha}(t) x^{\alpha}(\sigma(t))} \\
& +\frac{v(t)}{x^{\alpha}(t)}\left(p(t)\left(x^{\Delta}(t)\right)^{\alpha}-q(t) f(x(t))\right) \\
= & -\frac{v(t) q(t) f(x(t))}{x^{\alpha}(t)}+v(t) \frac{p(t)\left(x^{\Delta}(t)\right)^{\alpha}}{x^{\alpha}(t)}+\frac{v^{\Delta}(t)}{v(\sigma(t))} w(\sigma(t)) \\
& -\frac{v(t) r(\sigma(t))\left(x^{\Delta}(\sigma(t))\right)^{\alpha}\left(x^{\alpha}(t)\right)^{\Delta}}{x^{\alpha}(t) x^{\alpha}(\sigma(t))}
\end{aligned}
$$

Using (4) in (6) and by (H3), we have

$$
\begin{aligned}
w^{\Delta}(t) \leqslant & -k v(t) q(t)+v(t) \frac{p(t) r(\sigma(t))\left(x^{\Delta}(\sigma(t))\right)^{\alpha}}{r(t) x^{\alpha}(\sigma(t))}+\frac{v^{\Delta}(t)}{v(\sigma(t))} w(\sigma(t)) \\
& -\frac{v(t) r(\sigma(t))\left(x^{\Delta}(\sigma(t))\right)^{\alpha}\left(x^{\alpha}(t)\right)^{\Delta}}{x^{\alpha}(t) x^{\alpha}(\sigma(t))} \\
= & -k v(t) q(t)+\frac{\psi(t)}{v(\sigma(t))} w(\sigma(t))-\frac{v(t) r(\sigma(t))\left(x^{\Delta}(\sigma(t))\right)^{\alpha}\left(x^{\alpha}(t)\right)^{\Delta}}{x^{\alpha}(t) x^{\alpha}(\sigma(t))}
\end{aligned}
$$

where $\psi(t)$ as defined as (3).

By Lemma 2.5, if $0<\alpha \leqslant 1$, we have

$$
\begin{aligned}
w^{\Delta}(t) & \leqslant-k v(t) q(t)+\frac{\psi(t)}{v(\sigma(t))} w(\sigma(t))-\frac{\alpha v(t) r(\sigma(t))\left(x^{\Delta}(\sigma(t))\right)^{\alpha} x^{\Delta}(t)}{x^{\alpha}(t) x(\sigma(t))} \\
& \leqslant-k v(t) q(t)+\frac{\psi(t)}{v(\sigma(t))} w(\sigma(t))-\frac{\alpha v(t) r(\sigma(t))\left(x^{\Delta}(\sigma(t))\right)^{\alpha} x^{\Delta}(t)}{x^{\alpha+1}(\sigma(t))}
\end{aligned}
$$


if $\alpha>1$, we have

$$
\begin{aligned}
w^{\Delta}(t) & \leqslant-k v(t) q(t)+\frac{\psi(t)}{v(\sigma(t))} w(\sigma(t))-\frac{\alpha v(t) r(\sigma(t))\left(x^{\Delta}(\sigma(t))\right)^{\alpha} x^{\Delta}(t)}{x(t) x^{\alpha}(\sigma(t))} \\
& \leqslant-k v(t) q(t)+\frac{\psi(t)}{v(\sigma(t))} w(\sigma(t))-\frac{\alpha v(t) r(\sigma(t))\left(x^{\Delta}(\sigma(t))\right)^{\alpha} x^{\Delta}(t)}{x^{\alpha+1}(\sigma(t))} .
\end{aligned}
$$

Thus, by (7) and (8), we obtain

$$
\begin{aligned}
w^{\Delta}(t) & \leqslant-k v(t) q(t)+\frac{\psi(t)}{v(\sigma(t))} w(\sigma(t))-\frac{\alpha v(t) r(\sigma(t))\left(x^{\Delta}(\sigma(t))\right)^{\alpha} x^{\Delta}(t)}{x^{\alpha+1}(\sigma(t))} \\
& =-k v(t) q(t)+\frac{\psi(t)}{v(\sigma(t))} w(\sigma(t))-\frac{\alpha v(t) r^{\frac{1}{\alpha}}(t) x^{\Delta}(t)}{v(\sigma(t)) x(\sigma(t)) r^{\frac{1}{\alpha}}(t)} w(\sigma(t)) \\
& \leqslant-k v(t) q(t)+\frac{\psi(t)}{v(\sigma(t))} w(\sigma(t))-\frac{\alpha v(t) r^{\frac{1}{\alpha}}(\sigma(t)) x^{\Delta}(\sigma(t))}{v(\sigma(t)) x(\sigma(t)) r^{\frac{1}{\alpha}}(t)} w(\sigma(t)) \\
& =-k v(t) q(t)+\frac{\psi(t)}{v(\sigma(t))} w(\sigma(t))-\frac{\alpha v(t)}{v^{\frac{\alpha+1}{\alpha}}(\sigma(t)) r^{\frac{1}{\alpha}}(t)} w^{\frac{\alpha+1}{\alpha}}(\sigma(t))
\end{aligned}
$$

hold for all $\alpha>0$.

Then, using the inequality [19]

$$
B u-C u^{\frac{1+\alpha}{\alpha}} \leqslant \frac{\alpha^{\alpha}}{(1+\alpha)^{\alpha+1}} \frac{B^{\alpha+1}}{C^{\alpha}},
$$

let $B=\frac{\psi(t)}{v(\sigma(t))}, C=\frac{\alpha v(t)}{v^{\frac{\alpha+1}{\alpha}}(\sigma(t)) r^{\frac{1}{\alpha}}(t)}$ and $u=w(\sigma(t))$, we obtain

$$
\begin{aligned}
w^{\Delta}(t) & \leqslant-k v(t) q(t)+\frac{\alpha^{\alpha}}{(1+\alpha)^{1+\alpha}}\left(\frac{\psi(t)}{v(\sigma(t))}\right)^{\alpha+1}\left(\frac{v^{\frac{\alpha+1}{\alpha}}(\sigma(t)) r^{\frac{1}{\alpha}}(t)}{\alpha v(t)}\right)^{\alpha} \\
& =-k v(t) q(t)+\frac{\psi^{\alpha+1}(t) r(t)}{(\alpha+1)^{\alpha+1} v^{\alpha}(t)} .
\end{aligned}
$$

Integrating (10) from $t_{3}$ to $t$, we obtain

$$
w(t)-w\left(t_{3}\right) \leqslant-\int_{t_{3}}^{t}\left(k v(s) q(s)-\frac{\psi^{\alpha+1}(s) r(s)}{(\alpha+1)^{\alpha+1} v^{\alpha}(s)}\right) \Delta s,
$$

which yields

$$
\int_{t_{3}}^{t}\left(k v(s) q(s)-\frac{\psi^{\alpha+1}(s) r(s)}{(\alpha+1)^{\alpha+1} v^{\alpha}(s)}\right) \Delta s \leqslant w\left(t_{3}\right)-w(t)<w\left(t_{3}\right)
$$

for all large $t$. This is contrary to (2).

Next, we consider Case(2). Then exists $t_{2} \geqslant t_{1}$ such that $\left(x^{\Delta}(t)\right)^{\alpha}<0$ for $t \geqslant t_{2}$. Define the function $u(t)=-r(t)\left(x^{\Delta}(t)\right)^{\alpha}$. Then from (1), we have

$$
u^{\Delta}(t)-\frac{p(t)}{r(t)} u(t) \geqslant 0 .
$$


Thus

so that

$$
u(t) \geqslant u\left(t_{2}\right) e_{\ominus \frac{p(t)}{r(t)}}\left(t, t_{2}\right)
$$

i.e.

$$
\left(x^{\Delta}(t)\right)^{\alpha} \leqslant-u\left(t_{2}\right)\left(\frac{1}{r(t)} e_{\ominus \frac{p(t)}{r(t)}}\left(t, t_{2}\right)\right)
$$

$$
x^{\Delta}(t) \leqslant\left(-u\left(t_{2}\right)\left(\frac{1}{r(t)} e_{\ominus \frac{p(t)}{r(t)}}\left(t, t_{2}\right)\right)\right)^{\frac{1}{\alpha}} .
$$

Integrating (11) from $t_{2}$ to $t$, we get

$$
x(t)-x\left(t_{2}\right) \leqslant\left(r\left(t_{2}\right)\right)^{\frac{1}{\alpha}} x^{\Delta}\left(t_{2}\right) \int_{t_{2}}^{t}\left(\frac{1}{r(s)} e_{\ominus \frac{p(m)}{r(m)}}\left(s, t_{2}\right)\right)^{\frac{1}{\alpha}} \Delta s .
$$

Condition (H4) implies that $x(t)$ is eventually negative, which is a contradiction. The proof is complete.

Corollary 3.2. Assume that (H1)-(H4) hold. If

$$
\limsup _{t \rightarrow \infty} \int_{t_{0}}^{t}\left(k q(s)-\frac{p^{\alpha+1}(s)}{(\alpha+1)^{\alpha+1} r^{\alpha}(t)}\right) \Delta s=\infty,
$$

the every solution of (1) is oscillatory.

Corollary 3.3. Assume that (H1)-(H4) hold. If there is $\lambda \geqslant 1$ such that

$$
\limsup _{t \rightarrow \infty} \int_{t_{0}}^{t}\left(k s^{\lambda} q(s)-\frac{\left(r(s)\left(s^{\lambda}\right)^{\Delta}-s^{\lambda} p(s)\right)^{\alpha+1}}{(\alpha+1)^{\alpha+1}\left(s^{\lambda}\right)^{\alpha} r^{\alpha}(s)}\right) \Delta s=\infty,
$$

the every solution of (1) is oscillatory.

Corollary 3.4. Assume that (H1)-(H4) hold. If

$$
\limsup _{t \rightarrow \infty} \int_{t_{0}}^{t}\left(k R\left(s, t_{0}\right) q(s)-\frac{\left(r(s)\left(R\left(s, t_{0}\right)\right)^{\Delta}-R\left(s, t_{0}\right) p(s)\right)^{\alpha+1}}{(\alpha+1)^{\alpha+1}\left(R\left(s, t_{0}\right)\right)^{\alpha} r^{\alpha}(s)}\right) \Delta s=\infty,
$$

where $R\left(t, t_{0}\right)=\int_{t_{0}}^{t} \frac{1}{r(s)} \Delta s$, the every solution of (1) is oscillatory.

Theorem 3.5. Assume that (H1)-(H4) hold. Furthermore, suppose that $v(t)$ be as defined in Theorem 3.1 and a function $H \in C(\mathbb{D}, \mathbb{T})$, where $\mathbb{D}:=\{(t, s): t \geqslant$ $\left.s \geqslant t_{0}\right\}$, such that

$$
\begin{aligned}
& H(t, t)=0, \quad \text { for } t \geqslant t_{0}, \\
& H(t, s)>0, \quad \text { for }(t, s) \in \mathbb{D}_{0},
\end{aligned}
$$

where $\mathbb{D}_{0}:=\left\{(t, s): t>s \geqslant t_{0}\right\}$, and $H$ has a nonpositive continuous partial derivative $H^{\Delta_{s}}(t, s):=\partial H(t, s) / \partial s$ on $\mathbb{D}_{0}$ with respect to the second variable and satisfies

$$
\limsup _{t \rightarrow \infty} \frac{1}{H\left(t, t_{0}\right)} \int_{t_{0}}^{t}\left(k H(t, s) v(s) q(s)-\frac{v^{\alpha+1}(\sigma(t)) r(s) A^{\alpha+1}(t, s)}{(1+\alpha)^{1+\alpha} H^{\alpha}(t, s) v^{\alpha}(s)}\right) \Delta s=\infty,
$$


where

$$
A(t, s)=H(t, s) \frac{\psi(s)}{v(\sigma(t))}+H^{\Delta_{s}}(t, s) .
$$

Then every solution of (1) is oscillatory.

Proof. Suppose to the contrary that $x(t)$ is a nonoscillatory solution of (11) and let $t_{1} \geqslant t_{0}$ be such that $x(t) \neq 0$ for all $t \geqslant t_{1}$, so with loss of generality, we may assume that $x(t)$ is an eventually positive solution of (1) with $x(t)>0$ for $t \geqslant t_{1}$ sufficiently large. In view of Theorem 3.1 we see that $x^{\Delta}(t)$ is eventually negative or eventually positive. If $x^{\Delta}(t)$ is eventually negative, we are then back to Case (2) of Theorem 3.1 and we obtain a contradiction.

If $x^{\Delta}(t)$ is eventually positive, we assume that there exists $t_{2}>t_{1}$ such that $x^{\Delta}(t) \geqslant 0$ for $t_{2} \geqslant t_{1}$ and proceed as in the proof of Case (1) of Theorem 3.1 and get (9). From (9), it follows that

$$
\begin{aligned}
\int_{t_{2}}^{t} k H(t, s) v(s) q(s) \Delta s \leqslant & -\int_{t_{2}}^{t} H(t, s) w^{\Delta}(s) \Delta s+\int_{t_{2}}^{t} H(t, s) \frac{\psi(s)}{v(\sigma(s))} w(\sigma(s)) \Delta s \\
& -\int_{t_{2}}^{t} H(t, s) \frac{\alpha v(s)}{v^{\frac{\alpha+1}{\alpha}}(\sigma(s)) r^{\frac{1}{\alpha}}(s)} w^{\frac{\alpha+1}{\alpha}}(\sigma(s)) \Delta s
\end{aligned}
$$

Using the integration by parts formula, we have

$$
\begin{aligned}
\int_{t_{2}}^{t} H(t, s) w^{\Delta}(s) \Delta s & =\left.H(t, s) w(s)\right|_{t_{2}} ^{t}-\int_{t_{2}}^{t} H^{\Delta_{s}}(t, s) w(\sigma(s)) \Delta s \\
& =-H\left(t, t_{2}\right) w\left(t_{2}\right)-\int_{t_{2}}^{t} H^{\Delta_{s}}(t, s) w(\sigma(s)) \Delta s,
\end{aligned}
$$

where $H(t, t)=0$. Substituting (14) into (13), we obtain

$$
\begin{aligned}
& \int_{t_{2}}^{t} k H(t, s) v(s) q(s) \Delta s \leqslant \\
& H\left(t, t_{2}\right) w\left(t_{2}\right)+\int_{t_{2}}^{t} H^{\Delta_{s}}(t, s) w(\sigma(s)) \Delta s+\int_{t_{2}}^{t} H(t, s) \frac{\psi(s)}{v(\sigma(s))} w(\sigma(s)) \Delta s \\
& -\int_{t_{2}}^{t} H(t, s) \frac{\alpha v(s)}{v^{\frac{\alpha+1}{\alpha}}(\sigma(s)) r^{\frac{1}{\alpha}}(s)} w^{\frac{\alpha+1}{\alpha}}(\sigma(s)) \Delta s .
\end{aligned}
$$

Hence,

$$
\begin{aligned}
& \int_{t_{2}}^{t} k H(t, s) v(s) q(s) \Delta s \leqslant \\
& H\left(t, t_{2}\right) w\left(t_{2}\right)+\int_{t_{2}}^{t}\left(H(t, s) \frac{\psi(s)}{v(\sigma(s))}+H^{\Delta_{s}}(t, s)\right) w(\sigma(t)) \Delta s \\
& -\int_{t_{2}}^{t} H(t, s) \frac{\alpha v(s)}{v^{\frac{\alpha+1}{\alpha}}(\sigma(s)) r^{\frac{1}{\alpha}}(s)} w^{\frac{\alpha+1}{\alpha}}(\sigma(t)) \Delta s .
\end{aligned}
$$


Then, using the inequality [19]

$$
B u-C u^{\frac{1+\alpha}{\alpha}} \leqslant \frac{\alpha^{\alpha}}{(1+\alpha)^{\alpha+1}} \frac{B^{\alpha+1}}{C^{\alpha}},
$$

let $B=H(t, s) \frac{\psi(t)}{v(\sigma(t))}, C=H(t, s) \frac{\alpha v(t)}{v^{\frac{\alpha+1}{\alpha}}(\sigma(t)) r^{\frac{1}{\alpha}}(t)}$ and $u=w(\sigma(t))$, we obtain

$$
\int_{t_{2}}^{t} k H(t, s) v(s) q(s) \Delta s \leqslant H\left(t, t_{2}\right) w\left(t_{2}\right)+\int_{t_{2}}^{t} \frac{v^{\alpha+1}(\sigma(t)) r(s) A^{\alpha+1}(t, s)}{(1+\alpha)^{1+\alpha} H^{\alpha}(t, s) v^{\alpha}(s)} \Delta s .
$$

Then for all $t \geqslant t_{2}$, we have

$$
\int_{t_{2}}^{t}\left(k H(t, s) v(s) q(s)-\frac{v^{\alpha+1}(\sigma(t)) r(s) A^{\alpha+1}(t, s)}{(1+\alpha)^{1+\alpha} H^{\alpha}(t, s) v^{\alpha}(s)}\right) \Delta s \leqslant H\left(t, t_{2}\right) w\left(t_{2}\right),
$$

and this implies that

$$
\frac{1}{H\left(t, t_{2}\right)} \int_{t_{2}}^{t}\left(k H(t, s) v(s) q(s)-\frac{v^{\alpha+1}(\sigma(t)) r(s) A^{\alpha+1}(t, s)}{(1+\alpha)^{1+\alpha} H^{\alpha}(t, s) v^{\alpha}(s)}\right) \Delta s \leqslant w\left(t_{2}\right)
$$

for all large $t$, which contradicts (12). The proof is complete.

\section{Examples}

Example 4.1. Consider the equation

$$
\left(\left(x^{\Delta}(t)\right)^{\alpha}\right)^{\Delta}+\frac{1}{t}\left(x^{\Delta}(t)\right)^{\alpha}+t x^{\alpha}(t)=0,
$$

where $r(t)=1, \quad p(t)=-\frac{1}{t}, \quad q(t)=t, \quad \mu(t)=\frac{t}{2}, \quad f(x(t))=x^{\alpha}(t)$ with $k=1$, and $\alpha>0$. It is clear that conditions (H1)-(H4) are satisfied. Letting $v(t)=t$, $\mathbb{T}=[1, \infty)$, by Lemma 2.3 and Lemma 2.4 we have

$$
\begin{gathered}
e_{\frac{p}{r}}\left(t, t_{0}\right)=e_{-\frac{1}{t}}(t, 1)=\exp \left(\int_{1}^{t} \xi_{0}\left(-\frac{1}{\tau}\right) d \tau\right) \\
=\exp \left(\int_{1}^{t}\left(-\frac{1}{\tau}\right) d \tau\right)=\frac{1}{t} \\
e_{\ominus \frac{p}{r}}\left(t, t_{0}\right)=\frac{1}{e_{\frac{p}{r}}\left(t, t_{0}\right)}=t \\
\int_{t_{0}}^{\infty}\left(\frac{1}{r(t)} e_{\ominus \frac{p}{r}}\left(t, t_{0}\right)\right)^{\frac{1}{\alpha}} \Delta t=\int_{t_{0}}^{\infty}(t)^{\frac{1}{\alpha}} \Delta t=\infty, \\
\psi(t)=v^{\Delta}(t)+v(t) p(t)=0 .
\end{gathered}
$$

Hence,

$$
\limsup _{t \rightarrow \infty} \int_{t_{0}}^{t}\left(k v(s) q(s)-\frac{\psi^{\alpha+1}(s) r(s)}{(\alpha+1)^{\alpha+1} v^{\alpha}(s)}\right) \Delta s=\limsup _{t \rightarrow \infty} \int_{t_{0}}^{t}\left(t^{2}\right) \Delta s=\infty .
$$

That is (2) holds. By Theorem 3.1 we see that (15) is oscillatory. 


\section{Conclusion}

The results of this article are presented in a form which is essentially new and of high degree of generalize. In this article, using generalized Riccati transformation and inequality technique, we offer some new sufficient conditions which insure that any solution of dynamic equation (11) oscillates. In addition, we can try to get some oscillation behavior of dynamic equation (11) if $q(t)<0$ or $\int_{t_{0}}^{\infty}\left(\frac{1}{r(t)} e_{\ominus \frac{p(s)}{r(s)}}\left(t, t_{0}\right)^{\frac{1}{\alpha}} \Delta t<\infty\right.$ in the future work.

\section{Acknowledgments}

This research is supported by Shandong Provincial Natural Science Foundation ( ZR2017MA043).

\section{Competing Interests}

The authors do not have any competing interests in the manuscript.

\section{REFERENCES}

1. Hilger, S. (1990). Analysis on measure chainsa unified approach to continuous and discrete calculus. Results in Mathematics, 18(1-2), 18-56.

2. Bohner, M., \& Peterson, A. Dynamic Equations on Time Scales: An Introduction with Applications. 2001.

3. Bohner, M., \& Peterson, A. C. (Eds.). (2002). Advances in dynamic equations on time scales. Springer Science \& Business Media.

4. Erbe, L. (2001). Oscillation criteria for second order linear equations on a time scale. Canad. Appl. Math. Quart, 9(4), 345-375.

5. Sahi, Y. (2005). Oscillation of second-order delay differential equations on time scales. Nonlinear Analysis: Theory, Methods \&5 Applications, 63(5-7), e1073-e1080.

6. Saker, S. H. (2006). Oscillation of second-order nonlinear neutral delay dynamic equations

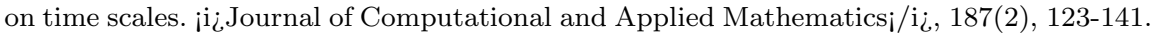

7. Han, Z., Sun, S., \& Shi, B. (2007). Oscillation criteria for a class of second-order EmdenFowler delay dynamic equations on time scales. Journal of Mathematical Analysis and Applications, 334(2), 847-858.

8. Hassan, T. S., Erbe, L., \& Peterson, A. (2010). Oscillation of second order superlinear dynamic equations with damping on time scales. Computers $\&$ Mathematics with Applications, 59(1), 550-558.

9. Shi, Y., Han, Z., \& Hou, C. (2017). Oscillation criteria for third order neutral EmdenFowler delay dynamic equations on time scales. Journal of Applied Mathematics and Computing, 55(1-2), 175-190.

10. Sui, Y., \& Han, Z. (2017). Oscillation of third-order nonlinear delay dynamic equation with damping term on time scales. Journal of Applied Mathematics and Computing, 1-23.

11. Sui, Y., \& Sun, S. (2018). Oscillation of third order nonlinear damped dynamic equation with mixed arguments on time scales. Advances in Difference Equations, 2018(1), 1-17.

12. Saker, S. H. (2004). Oscillation of nonlinear dynamic equations on time scales. Applied Mathematics and Computation, 148(1), 81-91.

13. Hassan, T. S. (2008). Oscillation criteria for half-linear dynamic equations on time scales. Journal of Mathematical Analysis and Applications, 345(1), 176-185. 
14. Saker, S. H., Agarwal, R. P., \& O'Regan, D. (2007). Oscillation of second-order damped dynamic equations on time scales. Journal of Mathematical Analysis and Applications, 330(2), 1317-1337.

15. Erbe, L., Hassan, T. S., \& Peterson, A. (2008). Oscillation criteria for nonlinear damped dynamic equations on time scales. Applied Mathematics and Computation, 203(1), 343357.

16. Saker, S. H., \& Cheng, S. S. (2004). Oscillation criteria for difference equations with damping terms. Applied mathematics and computation, 148(2), 421-442.

17. Deng, X. H., Wang, Q. R., \& Zhou, Z. (2015). Oscillation criteria for second order nonlinear delay dynamic equations on time scales. Applied Mathematics and Computation, 269, 834840 .

18. Agwo, H. A., Khodier, A. M. M., \& Hassan, H. A. (2017). Oscillation criteria of second order half linear delay dynamic equations on time scales. Acta Mathematicae Applicatae Sinica, English Series, 1(33), 83-92.

19. Zhang, S. Y., \& Wang, Q. R. (2010). Oscillation of second-order nonlinear neutral dynamic equations on time scales. Applied Mathematics and Computation, 216(10), 2837-2848.

\section{Fanfan Li}

School of Mathematical Sciences, University of Jinan, Jinan, Shandong 250022, P R China. e-mail: lifanfan1994@163.com

Zhenlai Han

School of Mathematical Sciences, University of Jinan, Jinan, Shandong 250022, P R China. e-mail: hanzhenlai@163.com 